\title{
Synergistic Dispatching Model of Power Source and Electric Network Considering Transmission - Distribution Connection Topology Optimization
}

\author{
Huadong $\mathrm{Li}^{1}$, Jiayin Jiang ${ }^{2, \mathrm{a}}$, Si Yang ${ }^{3}$, Wenbo $\mathrm{Li}^{1, \mathrm{~b}, *}$, Zhi Li ${ }^{4}$, Ming $\mathrm{Li}^{4}$ \\ ${ }^{1}$ State Grid Shandong Electric Power Research Institute, Jinan, China \\ ${ }^{2}$ Key Laboratory of Power System Intelligent Dispatch and Control of Ministry of Education (Shandong \\ University), Jinan, China
}

${ }^{3}$ Economic \& Technology Research Institute, State Grid Shandong Electric Power Company, Jinan, China

${ }^{4}$ State Grid Shandong Electric Power Company, Jinan, China.

a jiayin0227@163.com, biwnebo_1984@foxmail.com

Keywords: Economic dispatching, synergy between power source and electric network, coordination between transmission and distribution network, topology optimization

Abstract. For the large-scale integration of renewable generation into power grid and strengthening of active load regulation ability, the power transmission and distribution network coupling has become closer, and the contradiction between the source and network has been more prominent, which has brought new challenges to power system operation dispatching. In order to further enhance the coordination and interaction ability of the power grid, a synergistic dispatching model of power source and electric network is constructed on the basis of the traditional economic dispatching. By optimizing the transmission-distribution network connection topology, the operation economy has improved while ensuring system reliability. Case studies on a realistic power system show that the mathematical model is advantageous to the optimization of the whole power grid operation.

\section{Introduction}

Since 1920, as the basic means of power system optimal operation, the traditional economic dispatching aims at meeting load demand efficiently and minimizing operation cost by optimizing the generation unit output in advance, based on the load forecast and fixed power network topology. After nearly a hundred years of development, the power system economic dispatching theory and practice has been mature [1-5].

In recent years, with the increasing attention on the energy and the environment issues, it has been a human consensus to develop the Global Energy Internet and achieve sustainable development. Due to the changes of power grid pattern, the dispatching operation has faced a series of challenges: 1) With the large-scale integration of renewable resources, massive curtailment occurs due to the randomness and fluctuation characteristics of renewable energy, and contradiction between the source and network will be further intensified; 2) The development of energy storage, electric vehicle and flexible load has switched the power grid dispatching from the "generation schedule traces the changes of the load" to the "power source-electric network-load interacts", which leads to a distributed trend; 3) The regional intelligent agents represented by the micro-grid and the active distribution network cannot only realize the optimal operation of the internal network, but can also coordinate with the whole power grid. The operation status of network equipment has been seen as decision variables in the economic dispatching to improve the efficiency of power system and realize synergy between power source and electric network [6-10]. Additionally, the concept of economic dispatching from transmission network economic dispatching and regional intelligent agents to the transmission-distribution network synergy [11-14], and provided an important theoretical foundation for the development of the Global Energy Internet.

It can be seen that achieving synergistic dispatching is the fundamental for the optimal operation 
of the future power grid. At present, the electric network topology optimization is mainly concentrated in optimal transmission switching (OTS) or distribution network reconstruction (DNR). While the power flow analysis, optimal dispatching and voltage support of the transmission and distribution synergy are mostly based on fixed transmission-distribution connection topology. In fact, most of the distribution substations are supplied with dual-supply and dual-loop electrical system, and the connection branch switching can be achieved through the remote control. In this way, the key indicators such as load mode, locational marginal prices (LMP) and voltage quality can be improved. This paper focuses on synergistic dispatching of power source and electric network, and proposes a mathematical model of economic dispatching considering transmissiondistribution connection topology optimization.

The paper is organized as follows. Section 2 introduces several typical transmission-distribution network connection topology. Section 3 presents the mathematical model for dynamic economic dispatching model with distribution network branch switching. In Section 4, case studies are conducts to illustrate the effectiveness and feasibility of the proposed method. Section 5 is the summary and prospect.

\section{Transmission-distribution connection redundancy topology architecture}

In the stage of power network planning, considering factors such as load demand, geographical location and line corridor, a variety of transmission-distribution network connection topology structures are formed. Several transmission and distribution connection topologies based on actual network in China are shown in Fig.1- Fig.5 respectively.

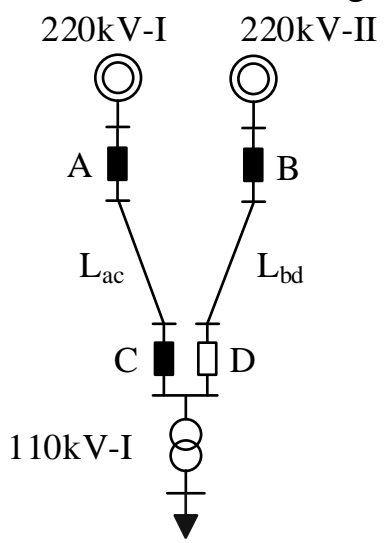

Fig.1 Dual-supply and dual-loop power supply of $110 \mathrm{kV}$ substation.

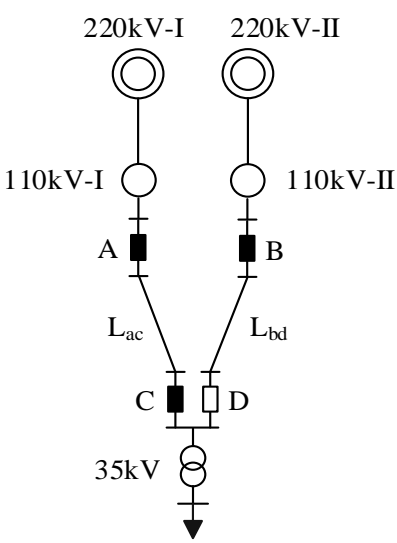

Fig.2 Dual-supply and dual-loop power supply of $35 \mathrm{kV}$ substation.

The dual-supply and dual-loop power supply of $110 \mathrm{kV}$ substation is shown in Fig.1. The substation $110 \mathrm{kV}$ station I can be connected to $220 \mathrm{kV}$ station I and $220 \mathrm{kV}$ station II through the branches $\mathrm{L}_{\mathrm{ac}}$ and $\mathrm{L}_{\mathrm{bd}}$ respectively by remotely switching $\mathrm{C}$ and $\mathrm{D}$. In case of normal operation, switch A and switch B are online, one of switch $C$ and switch D is online and the other is regarded as the hot standby. The two $220 \mathrm{kV}$ substations can be regarded as two independent power supplies for $110 \mathrm{kV}$ station I as they belong to different buses. Similarly, the schematic diagram for the typical 35kV substation dual-supply and dual-loop power supply is as shown in Fig.2. 


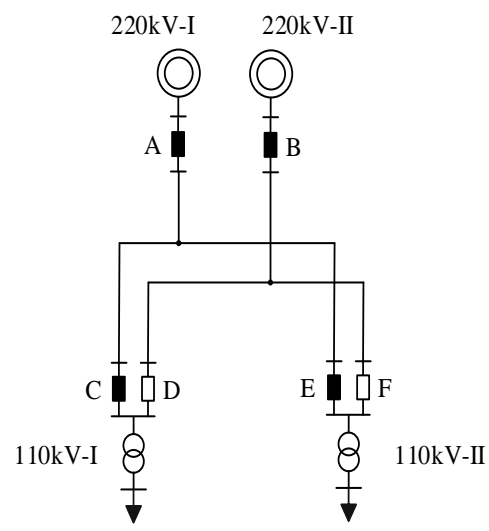

Fig.3 Dual-supply under T connection mode

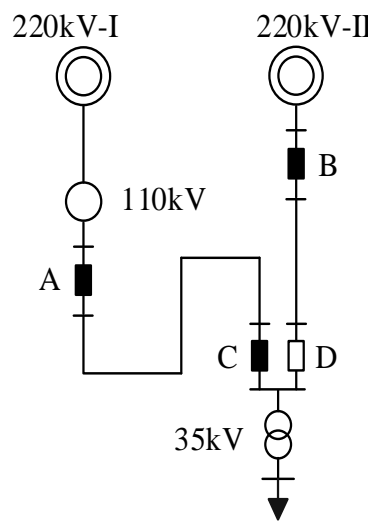

Fig.4 Distribution substation connect to substation at different voltage levels

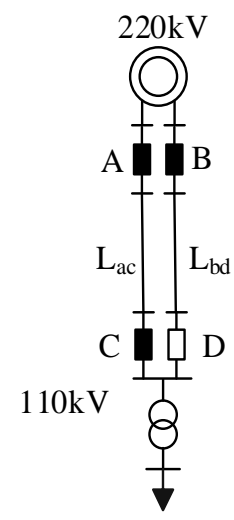

Fig.5 Single-supply and dualloop power supply of $110 \mathrm{kV}$ substations

Considering the practical limit of power grid planning, the completely independent power supply branches are difficult to achieve. In fact, T connection mode are more common as shown in Fig.3. Compared with Fig.1, although the branches are not directly connected to the superior substation, the online switching can still be achieved through controlling switches (switch $\mathrm{C}$ and switch D, switch E and switch F) at the substation side.

As shown in Fig.4, this power supply mode is applied to some $35 \mathrm{kV}$ substations that a branch is connected to $35 \mathrm{kV}$ bus of $110 \mathrm{kV}$ substation, and the other is connected to $35 \mathrm{kV}$ transformer side of $220 \mathrm{kV}$ substation. Normally, $220 \mathrm{kV}$ transformer is the three-winding transformer. Therefore, if $110 \mathrm{kV}$ transformer is the two-winding transformer, online switching can be achieved, otherwise, if $110 \mathrm{kV}$ transformer is the three-winding transformer, online operation is not allowed. The branch switching can only be operated after power cut of distribution substation.

In the realistic power grid, there are a few dual-loop power supply modes as shown in Fig.5. The distribution substation has two power supply branches that are from the same $220 \mathrm{kV}$ substation, which has no effect on the power flow of transmission network and optimal operation of power grid.

In summary, it can be known that transmission-distribution connection topology has inevitably effects on the power flow of transmission network. Optimizing the topology of the transmissiondistribution can achieve the synergy between power source and electric network, and improve operation efficiency of the power grid.

\section{Synergistic dispatching model of power source and electric network considering transmission-distribution connection topology optimization}

In order to illuminate the influence of the transmission-distribution connection topology on the power system optimal operation, this paper ignores the impact of voltage magnitude and network loss. Considering transmission-distribution connection topology optimization, based on DC power flow, synergistic dispatching model of power source and electric network is proposed.

\subsection{Objective function}

The objective of the traditional economic dispatching problem is to minimize the system operation cost. In this paper, the load balance degree is added to the objective function. The load balancing is important for safety of power systems [15], especially in the system with large-scale renewable generation integration. Under the guidance of load balance degree, the unique optimal solution will be found.

$$
\min \sum_{t \in T} \sum_{g \in G}\left(c_{n g} P_{n g, t}+b_{n g}\right)+K \cdot \sum_{t \in T} \sqrt{\sum_{j \in L}\left(R_{j}-\frac{\sum_{k \in L} R_{k}}{N}\right)^{2}}
$$


where $T, G$ and $L$ denote the sets of period, generators and branches respectively; $N$ is the total number of branches; $c_{n g}$ and $b_{n g}$ are the cost coefficients of generator $g ; P_{n g, t}$ is the active power injected into bus $n$ by generator $g$ in period $t$; $R_{k, t}$ is the load balance degree of the branch $k^{\text {th }}$ in period $t, R_{k, t}=P_{n k, t} / P_{n k m a x} ; P_{n k, t}$ is the active power flow on branch $k^{\text {th }}$ to bus $n$ in period $t$; $P_{n k m a x}$ is the maximum active power on branch $k^{\text {th }}$; $K$ is the proportional coefficient of objective function.

\subsection{Constraint conditions}

\subsubsection{Generator active power constraint}

$$
P_{n g}^{\min } \leq P_{n g, t} \leq P_{n g}^{\max }
$$

where $P_{n g}^{\min }$ and $P_{n g}^{\max }$ are the minimum and maximum of active power injected into the bus $n$ by the generator $g$ respectively.

\subsubsection{Voltage angle constraint}

$$
\theta_{n}^{\min } \leq \theta_{n, t} \leq \theta_{n}^{\max }
$$

where $\theta_{n, t}$ is the voltage angle of bus $n$ in period $t ; \theta_{n}^{\min }$ and $\theta_{n}^{\max }$ are the minimum and maximum voltage angle of bus $n$ in period $t$ respectively.

\subsubsection{Nodal power balance constraint}

$$
\sum_{k \in L} P_{n k, t}+\sum_{g \in G} P_{n g, t}=\sum_{d \in D} P_{n d, t}
$$

where $D$ denotes the set of buses; $P_{n d, t}$ denotes the active power load at bus $n$ in period $t$.

\subsubsection{Branch transmission power constraint}

$$
P_{n k}^{\min } Z_{k, t} \leq P_{n k, t} \leq P_{n k}^{\max } Z_{k, t}
$$

where $P_{n k}^{\min }$ and $P_{n k}^{\max }$ are the minimum and maximum active power on branch $k^{\text {th }} ; z_{k, t}$ represents the status of the branch $k^{\text {th }}$ that $z_{k, t}=1$ reflects a closed status, and $z_{k, t}=0$ reflects an open status.

\subsubsection{Branch power flow balance constraint}

In the proposed model, we assume that the transmission network topology has been fixed. Thus, the branch power flow balance constraint need to be satisfied for transmission branches.

$$
B_{k}\left(\theta_{n, t}-\theta_{m, t}\right)-P_{n k, t}=0 \quad k \in L T
$$

where $L T$ is the set of transmission branches; $B_{k}$ is the electrical susceptance of the branch $k^{\text {th }} ; n$ and $m$ are the buses at two ends of the branch $k^{\text {th }}$.

The branch power flow balance constraints for the distribution substation branches are as shown in formula (7). The branch status variable $z_{k, t}=1$ reflects that two constraints are transformed into an equation. If the variable $z_{k, t}=0$, the formula (7) will be transformed into two unconstrained inequalities due to the large value $M$.

$$
\begin{aligned}
& B_{k}\left(\theta_{n, t}-\theta_{m, t}\right)-P_{n k, t}+\left(1-z_{k, t}\right) M \geq 0 \\
& B_{k}\left(\theta_{n, t}-\theta_{m, t}\right)-P_{n k, t}-\left(1-z_{k, t}\right) M \leq 0
\end{aligned} \quad k \in L D
$$

where $L D$ is the set of distribution substation branches; $M$ is a given large value which takes $B_{k}\left(\theta_{n, t}^{\max }-\theta_{m, t}^{\min }\right)$ in this paper.

\subsubsection{Uniqueness constraint for power supply branch}

There is only one power supply branch for each distribution substation at bus $d$. The sum of the power supply branch status variables for bus d equal to 1.

$$
\sum_{k \in d K} z_{k, t}=1
$$


where $d K$ is the set of power supplying branches to the distribution substation at bus $d$.

\section{Case study}

To illustrate the effectiveness of the proposed mathematical model, a 6-bus transmission system and actual transmission-distribution connection topology in Shandong province is considered. The 6-bus transmission system is as shown in Fig.6.

The parameters of branch and generator are listed in Table 1 and Table 2, the transmissiondistribution network connection topology architecture is as shown in Table 3, and load curve at four time periods are shown in Fig.7.

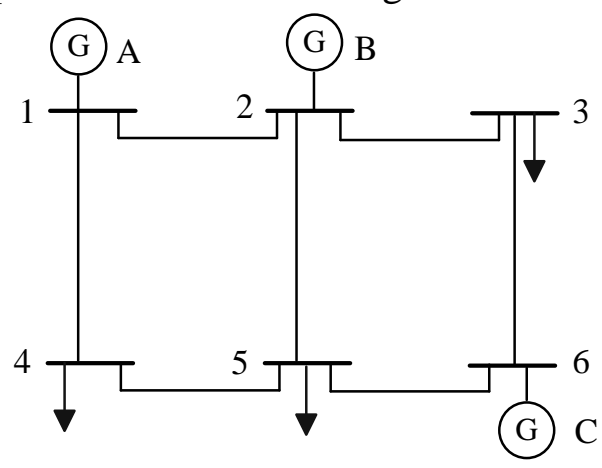

Fig.6 6-bus transmission system diagram

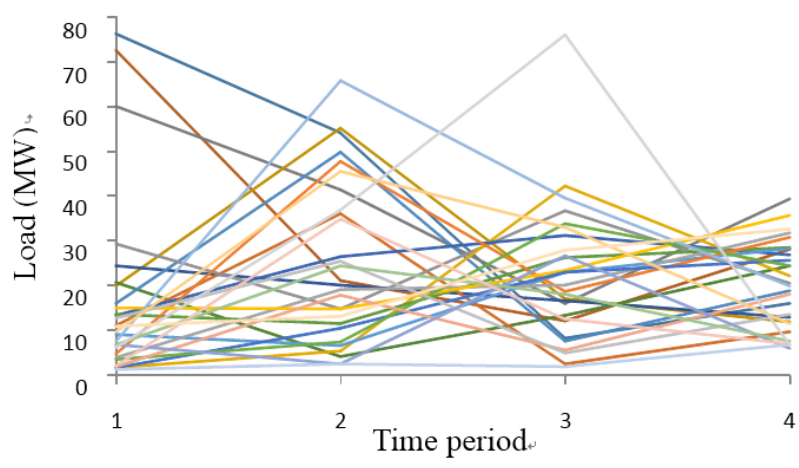

Fig.7 Load curve of the actual power system

Table 1 Branch parameters for the 6-bus system

\begin{tabular}{|c|c|c|}
\hline Transmission branch & $\begin{array}{c}\text { Reactance } \\
(\mathrm{pu})\end{array}$ & $\begin{array}{c}\text { Transmission capacity } \\
(\mathrm{MW})\end{array}$ \\
\hline $1-2$ & 0.17 & 200 \\
\hline $1-4$ & 0.258 & 300 \\
\hline $2-3$ & 0.037 & 300 \\
\hline $2-5$ & 0.197 & 100 \\
\hline $3-6$ & 0.018 & 200 \\
\hline $4-5$ & 0.037 & 200 \\
\hline $5-6$ & 0.14 & 200 \\
\hline
\end{tabular}

Table 2 Generator parameters for the 6-bus system

\begin{tabular}{|c|c|c|}
\hline Generator & $\begin{array}{c}\text { Capacity } \\
\text { (MW) }\end{array}$ & $\begin{array}{c}\text { Marginal cost } \\
\text { (USD/MWh) }\end{array}$ \\
\hline A & 300 & 20 \\
\hline B & 200 & 29 \\
\hline C & 500 & 40 \\
\hline
\end{tabular}


Table 3 Transmission-distribution network connection topology

\begin{tabular}{|c|c|c|c|}
\hline Distribution bus & $\begin{array}{c}\text { Superior } \\
\text { connection bus }\end{array}$ & $\begin{array}{c}\text { Distribution } \\
\text { bus }\end{array}$ & $\begin{array}{c}\text { Superior } \\
\text { related bus }\end{array}$ \\
\hline 7 & 3,4 & 20 & 18,21 \\
\hline 8 & 3,4 & 21 & 11 \\
\hline 9 & $3,4,5$ & 22 & 3 \\
\hline 10 & 3,5 & 23 & 5 \\
\hline 11 & 3,5 & 24 & 5 \\
\hline 12 & 3,5 & 25 & 4 \\
\hline 13 & 4,5 & 26 & 4 \\
\hline 14 & 4,5 & 27 & 4 \\
\hline 15 & 4,5 & 28 & 3 \\
\hline 16 & 8,15 & 29 & 3 \\
\hline 17 & 14,10 & 30 & - \\
\hline 18 & 10,11 & 31 & - \\
\hline 19 & 11,12 & - & \\
\hline
\end{tabular}

Two economic dispatch schemes are carried out as follow.

Scheme I: Economic dispatch is carried out based on the fixed transmission-distribution connection topology designated at random during the study period.

Scheme II: Economic dispatch is carried out considering transmission-distribution connection topology optimization.

Table 4 Fixed transmission-distribution connection topology in Scheme I

\begin{tabular}{|c|c|c|c|c|c|}
\hline Distribution bus & $\begin{array}{c}\text { Connection } \\
\text { branch }\end{array}$ & $\begin{array}{c}\text { Branch } \\
\text { status }\end{array}$ & Distribution bus & $\begin{array}{c}\text { Connection } \\
\text { branch }\end{array}$ & $\begin{array}{c}\text { Branch } \\
\text { status }\end{array}$ \\
\hline \multirow{2}{*}{7} & $3-7$ & 0 & \multirow{2}{*}{14} & $4-14$ & 0 \\
\hline & $4-7$ & 1 & & $5-14$ & 1 \\
\hline \multirow{2}{*}{8} & $3-8$ & 1 & \multirow{2}{*}{15} & $4-15$ & 0 \\
\hline & $4-8$ & 0 & & $5-15$ & 1 \\
\hline \multirow{2}{*}{9} & $3-9$ & 1 & \multirow{2}{*}{16} & $8-16$ & 1 \\
\hline & $4-9$ & 0 & & $15-16$ & 0 \\
\hline \multirow{2}{*}{10} & $3-10$ & 0 & \multirow{2}{*}{17} & $10-17$ & 0 \\
\hline & $5-10$ & 1 & & $14-17$ & 1 \\
\hline \multirow{2}{*}{11} & $3-11$ & 0 & \multirow{2}{*}{18} & $10-18$ & 1 \\
\hline & $5-11$ & 1 & & $11-18$ & 0 \\
\hline \multirow{2}{*}{12} & $3-12$ & 1 & \multirow{2}{*}{19} & $11-19$ & 1 \\
\hline & $5-12$ & 0 & & $12-19$ & 0 \\
\hline \multirow{2}{*}{13} & $4-13$ & 0 & \multirow{2}{*}{20} & $18-20$ & 1 \\
\hline & $5-13$ & 1 & & $21-20$ & 0 \\
\hline
\end{tabular}

Note: 1 is the closed state, 0 is the disconnected state

Table 5 Optimal generator output results in Scheme I

\begin{tabular}{|c|c|c|c|c|}
\hline Time period & $\mathrm{t} 1$ & $\mathrm{t} 2$ & $\mathrm{t} 3$ & $\mathrm{t} 4$ \\
\hline Unit A (MW) & 300.0 & 300.0 & 259.2 & 300.0 \\
\hline Unit B (MW) & 82.9 & 0.0 & 0.0 & 0.0 \\
\hline Unit C (MW) & 82.9 & 328.2 & 360.8 & 270.8 \\
\hline Total cost (USD) & \multicolumn{5}{|c|}{67299.5} \\
\hline Load balance degree & \multicolumn{5}{|c|}{19.01} \\
\hline
\end{tabular}

The fixed topology is shown in Table 4 and the optimization results of Scheme I are as shown in 
Table 5. Total operating cost is 67299.5USD and the balance degree is 19.01. It can be seen that the output of unit $\mathrm{C}$ is much more than the more economical unit $\mathrm{B}$. It is because that, with more distribution substations connected to bus 4 and 5, larger power flow passing through branch 2-5 which is of smaller transmission capacity and other branches with abundant transmission capacity cannot play a role.

Table 6 Optimal transmission-distribution connection topology results in Scheme II

\begin{tabular}{|c|c|c|c|c|c|c|c|c|c|c|c|}
\hline \multirow{2}{*}{\multicolumn{2}{|c|}{$\begin{array}{c}\text { Distribution bus } \\
\text { Connection branch }\end{array}$}} & \multicolumn{2}{|c|}{7} & \multicolumn{2}{|c|}{10} & \multicolumn{2}{|c|}{11} & \multicolumn{2}{|c|}{13} & \multicolumn{2}{|c|}{14} \\
\hline & & $3-7$ & $4-7$ & $3-10$ & $5-10$ & $3-11$ & $5-11$ & $4-13$ & $5-13$ & $4-14$ & $5-14$ \\
\hline \multirow{4}{*}{ Branch status } & $\mathrm{t} 1$ & 1 & 0 & 0 & 1 & 1 & 0 & 1 & 0 & 0 & 1 \\
\hline & $\mathrm{t} 2$ & 1 & 0 & 1 & 0 & 1 & 0 & 1 & 0 & 1 & 0 \\
\hline & $\mathrm{t} 3$ & 1 & 0 & 1 & 0 & 1 & 0 & 0 & 1 & 1 & 0 \\
\hline & $\mathrm{t} 4$ & 1 & 0 & 1 & 0 & 1 & 0 & 1 & 0 & 1 & 0 \\
\hline \multicolumn{2}{|c|}{ Distribution bus } & \multicolumn{2}{|c|}{15} & \multicolumn{2}{|c|}{17} & \multicolumn{2}{|c|}{18} & \multicolumn{2}{|c|}{19} & \multicolumn{2}{|c|}{20} \\
\hline \multicolumn{2}{|c|}{ Connection branch } & $4-15$ & $5-15$ & $10-17$ & $14-17$ & $10-18$ & $11-18$ & $11-19$ & $12-19$ & $18-20$ & $21-20$ \\
\hline \multirow{4}{*}{ Branch status } & $\mathrm{t} 1$ & 1 & 0 & 0 & 1 & 0 & 1 & 0 & 1 & 0 & 1 \\
\hline & $\mathrm{t} 2$ & 1 & 0 & 1 & 0 & 1 & 0 & 0 & 1 & 0 & 1 \\
\hline & t3 & 1 & 0 & 1 & 0 & 1 & 0 & 1 & 0 & 1 & 0 \\
\hline & $\mathrm{t} 4$ & 1 & 0 & 1 & 0 & 1 & 0 & 0 & 1 & 1 & 0 \\
\hline
\end{tabular}

Table 7 Optimal generator output results in Scheme II

\begin{tabular}{|c|c|c|c|c|}
\hline Time period & $\mathrm{t} 1$ & $\mathrm{t} 2$ & $\mathrm{t} 3$ & $\mathrm{t} 4$ \\
\hline Unit A (MW) & 300.0 & 300.0 & 300.0 & 300.0 \\
\hline Unit B (MW) & 165.8 & 196.5 & 200.0 & 199.5 \\
\hline Unit C (MW) & 0.0 & 221.0 & 120.0 & 116.4 \\
\hline Total cost (USD) & \multicolumn{3}{|c|}{64389.6} \\
\hline Load balance degree & \multicolumn{5}{|c|}{16.87} \\
\hline
\end{tabular}

The optimization results of Scheme II are as shown in Table 6 (only the topology connections different from Scheme I are listed) and Table 7. Total cost is reduced to 64389.6USD and the load balance degree is reduced to 16.87. By making full use of economic units and large-capacity transmission branches, the bottleneck effect caused by some weak parts has been alleviated.

It can be seen that the optimal power supply branches of the distribution buses are different due to the different load pattern. Considering the inconsistency of active distribution network and increase of distributed generation, it is necessary to seek for the optimal transmission-distribution connection topology.

\section{Summary and prospect}

In this paper, an economic dispatching model based on DC power flow is established by taking the transmission-distribution topology structure into the power grid optimization operation as decision variables. The positive effects of transmission-distribution coordination on power system economic dispatching has been illuminated.

Modern transmission network are connected with numerous distribution networks which are controlled by different subordinate control centres. For meeting the actual demand of the power grid, it is necessary to improve coordination between transmission and distribution network and enhance synergy between power source and electric network.

In addition, with the large-scale integration of renewable generation into distribution networks, the voltage fluctuations has become more and more serious. Further study need to be done based on the AC power flow. 


\section{References}

[1] WOOD A J, WOOLLENBERG B F. Power generation, operation, and control[M], Beijing: Tsinghua University Press, 2003.

[2] Carpentier J. Contribution a l' etude du dispatching economique[J]. Bulletin de la Societe Francaise des Electriciens, 1962, 3(8):431-447.

[3] Han Xueshan. Accumulation Variable Method of Dynamic Optimization Dispatching [D]. Harbin: Harbin Institute of Technology, 1994

[4] Zhang Boming, Wu Wenchuan, Zheng Taiyi. Design of a multi-time scale coordinated active power dispatching system for accommodating large scale wind power penetration[J]. Automation of Electric Power Systems, 2011,35(1):1-6.

[5] Zhong Shimin, Han Xushan, Liu Daowei. Benders decomposition algorithm for corrective security-constrained optimal power flow[J]. Proceedings of the CSEE, 2011, 31(1):65-71.

[6] Liu Guojing, HAN Xueshan, YANG Ming, LI Ming. Analysis and Verification on Three States Between Source and Network in Economic Dispatch. Power System Technology. 2013, 37(2): 431437

[7] Emily B.Fisher, Richard P.O’Neill, Michael C.Ferris. Optimal Transmission Swithching. IEEE Transactions on Power Systems, Vol.23, NO.3 AUGUST 2008:1346-1355

[8] Kory W.Hedman, Richard P.O’Neill, Emily Bartholomew Fisher, Shmuel S. Oren. Optimal Transmission Switching With Contingency Analysis. IEEE Transactions on Power Systems, Vol.24, NO.3, AUGUST 2009:1577-1586

[9] Kory W.Hedman, Michael C.Ferris, Richard P.O’Neill, Emily Bartholomew Fisher, Shmuel S. Oren. Co-Optimization of Generation Unit Commitment and Transmission Switching With N-1 Reliability:1052-1063

[10] Mojtaba Khanabadi, Hassan Ghasemi, Meysam Doostizadeh. Optimal Transmission Switching Considering Voltage Security and N-1 Contingency Analysis. IEEE Transactions on Power System, Vol.8, NO.1, FEBRUARY 2013:542-550

[11] Hongbin Sun, Qinglai Guo, Boming Zhang, Zhengshuo Li, Jianhui Wang. Master-SlaveSplitting Based Distributed Global Power Flow Method for Integrated Transmission and Distribution Analysis. IEEE Transactions on Smart Grid, Vol.6, NO.3, MAY 2015:1484-1492

[12] Zhenshuo Li, Qinglai Guo, Hongbin Sun, Jianhui Wang. Coordinated Transmission and Distribution AC Optimal Power Flow. IEEE Transactions on Smart Grid, 2016,pp(99):1-13

[13] Zhengshu Li, Jianhui Wang, Hongbin Sun, Qinglai Guo. Transmission Contingency Analysis Based on Integrated Transmission and Distribution Power Flow in Smart Grid. IEEE Transactions on Power Systems, VOL.30, NO.6, NOVEMBER 2015:3356-3367

[14] Zhenshuo Li, Jianhui Wang, Hongbin Sun, Qinglai Guo. Transmission Contingency Screening Considering Impacts of Distribution Grids. IEEE Transactions on Power Systems, Vol.31, NO.2, MARCH 2016:1659-1660

[15] Yu Haibo. Research of Power System Dispatching Strategy and Wind Power Planning Based on Load Rate Balance Degree. 2013, Harbin Institute of Technology. 